Check for updates

Cite this: Nanoscale Adv., 2019, 1, 3200

\title{
A bio-based piezoelectric nanogenerator for mechanical energy harvesting using nanohybrid of poly(vinylidene fluoride) $\dagger$
}

\author{
Anupama Gaur, (D) a Shivam Tiwari, (iD a Chandan Kumar iD b and Pralay Maiti (iD *a
}

A bio-based piezoelectric egg shell membrane (ESM) is used for energy harvesting applications in the form of two and three-component nanohybrids. A bio-waste piezo-filler in a piezoelectric polymer matrix was designed through an induced $\beta$-phase nucleation in the matrix using an organically modified twodimensional nanoclay. Structural alteration ( $\alpha$ to $\beta$-phase) in the presence of the nanoparticles was also manifested by morphological changes over spherulite to a needle-like morphology; thus, these nanohybrid materials are suitable for energy harvesting applications. ESM-based nanogenerators were fabricated with local ordering of piezo phases, as revealed via atomic force microscopy, leading to the generation of mostly electroactive phases in the whole nanohybrid. The voltage outputs from the optimized device were measured to be $\sim 56$ and $144 \mathrm{~V}$ in single and multiple stacks (five), respectively, with corresponding power densities of $55 \mu \mathrm{W} \mathrm{cm} \mathrm{cm}^{-2}$ and $100 \mu \mathrm{W} \mathrm{cm}{ }^{-2}$. The efficiency of the device was verified using a variety of body movements, e.g. bending, twisting, walking, and foot tapping, causing mechanical energy dissipation, which eventually transformed into energy storage. The underlying mechanism of high conversion of energy is explained by the synergistically induced piezo-phase in the polymer matrix together with the floppy piezo-filler. The mechanical stability, durability and repeated energy conversion of the hybrid device make it a robust nanogenerator. The biocompatibility of the nanogenerator was verified through cellular studies, demonstrating its appropriate use in powering biomedical devices/implants.

Received 5th April 2019

Accepted 26th June 2019

DOI: 10.1039/c9na00214f

rsc.li/nanoscale-advances

\section{Introduction}

With the decrease in available fuel and increase in environmental pollution from fossil fuel burning, it is urgent to find alternative green energy resources. ${ }^{1-4}$ Various alternative sources of energy have been developed, and energy harvesting is one of the efficient alternatives to reuse it in other form. Energy harvesting from mechanical waste energy is quite effective because it is ecological, sustainable and readily accessible., ${ }^{5,6}$ Mechanical energy from finger tapping, heel imparting, jogging, bending, joint movements, air flow, water flow, breathing and talking have been used for energy harvesting by several researchers. ${ }^{711}$ Self-powered devices or nanogenerators harvest the mechanical energy from different sources. To date, many approaches have been proposed based on triboelectric ${ }^{12}$ and piezoelectric ${ }^{13,14}$ nanogenerators. Triboelectric nanogenerators have high energy conversion efficiency and high

${ }^{a}$ School of Materials Science and Technology, Indian Institute of Technology (Banaras Hindu University), Varanasi 221005, India. E-mail: pmaiti.mst@itbhu.ac.in

${ }^{b}$ School of Biomedical Engineering, Indian Institute of Technology (Banaras Hindu University), Varanasi 221005, India

$\uparrow$ Electronic supplementary information (ESI) available. See DOI: $10.1039 /$ c9na00214f output voltage $;, 515$ however, they possess drawbacks such as low durability and warping problems due to their large size. Due to these problems, researchers are currently focusing on piezoelectric nanogenerators with mechanical durability, high performance and better sensitivity. ${ }^{6,716}$ Numerous materials have been used for piezoelectric nanogenerators, such as PZT, $\mathrm{ZnO}, \mathrm{BaTiO}_{3}, \mathrm{Na} / \mathrm{KNbO}_{3}$ and $\mathrm{ZnSnO}_{3}$ nanoparticles ${ }^{17-20}$ as well as certain polymers, such as poly(vinylidene fluoride) (PVDF), ${ }^{21}$ its copolymers with hexafluoropropylene $[\mathrm{P}(\mathrm{VDF}-\mathrm{HFP})]^{22}$ and trifluoroethylene $[\mathrm{P}(\mathrm{VDF}-\mathrm{TrFE})],{ }^{23}$ and poly(vinyl acetate) (PVAc). ${ }^{24}$ Many of these materials have limitations, such as brittleness, toxicity, non-biodegradability/non-biocompatibility and associated complex synthesis and fabrication processes. Although, polymers also have advantages, such as toughness, non-toxicity and biocompatibility; this benefits implants and biomedical health monitoring systems, which require devices to be biocompatible.

Biocompatible self-powered piezoelectric nanogenerators focus on common human body motions, particularly, in in vivo conditions, for health monitoring and safety purposes. ${ }^{25,26}$ Naturally abundant piezoelectric materials are suitable for the design of bio-medical nanogenerators or sensors, which can operate without any adverse effects on living systems. Natural piezoelectric materials such as hydroxyapatite, ${ }^{27}$ collagen 
fibrils, ${ }^{28,29}$ cellulose $^{30}$ and chitin $^{31}$ are suitable choices for designing biocompatible piezoelectric nanogenerators. Biobased piezoelectric materials are also used as energy harvesting materials. These materials have advantages such as nontoxicity and biocompatibility along with environmentally friendly behavior. A virus-based piezoelectric nanogenerator was fabricated; however, it showed a very low power density. ${ }^{26}$ The power generated using prawn shell ${ }^{31}$ and fish scale ${ }^{32}$ as biowaste was found to be 0.76 and $1.14 \mu \mathrm{W} \mathrm{cm}{ }^{-2}$, respectively, while bio-waste onion skin has been reported for electricity generation with a meager power density of $1.7 \mu \mathrm{W} \mathrm{cm}{ }^{-2} .^{33}$

Here, we used an egg shell membrane (ESM) as a piezoelectric filler for preparing nanohybrids of PVDF and twodimensional layered silicate (nanoclay) for energy harvesting. ESM also has many advantages: (i) it is inexpensive and readily available for use as industrial and household waste; (ii) it is nontoxic and environmentally friendly; (iii) it is composed of many proteins and amino acids having functional groups present on their surfaces, resulting in better functionalization capability; and (iv) it has weak chemical bonds, so it can be readily modified using carbonization and dissolution. Due to these advantages, ESM has been used in many fields, such as chemical, electrical, environmental and biomedical engineering. ${ }^{34}$ By adding ESM as a second filler in the nanohybrid, its energy harvesting capability was enhanced significantly along with its biocompatible nature, making it suitable for powering biomedical devices, including implants.

\section{Experimental}

\subsection{Materials}

Commercial poly(vinylidene fluoride) SOLEF 6008 with a molecular weight of $2.7 \times 10^{5}$ was kindly supplied by Ausimont, Italy. Organically modified clay, Cloisite $30 \mathrm{~B}$ (bis(hydroxyethyl) methyl tallow ammonium ion-exchanged montmorillonite), density $1.98 \mathrm{~g} \mathrm{~cm}^{-3}$, was purchased from Southern Clay Inc., USA. Dimethyl formamide (DMF), dimethyl sulfoxide (DMSO) and poly(dimethyl siloxane) (PDMS; Dow Corning Sylgard, 184 silicon elastomer) were purchased from Merck, Himedia and Ellsworth Adhesives, India, respectively. Chicken eggs were procured from a local market to obtain egg shells.

\subsection{Nanohybrid preparation}

Two types of nanohybrids were prepared using PVDF. One is a two-component system (PVDF + ESM) and the other is a threecomponent system (PVDF + nanoclay + egg shell). DMF was used as a common solvent for nanohybrid preparation. The egg was boiled and the inner soft layer covering the white part of the egg, i.e. the egg shell membrane (ESM), was peeled out carefully. The ESM was then dried in an oven and crushed into fine powder for use as a filler for nanohybrid preparation. PVDF was dissolved in DMF at $60{ }^{\circ} \mathrm{C}$, followed by the addition of crushed ESM to obtain the two-component nanohybrid. The resulting mixture was then stirred for $3 \mathrm{~h}$ and dried under reduced pressure to form a film; this nanohybrid is termed 'P-ESM'. For the three-component hybrids, first, $4 \mathrm{wt} \%$ nanoclay (with respect to polymer weight) was added to a PVDF solution. The crushed ESM was added in an appropriate quantity to the mixed solution, followed by ultrasonication to disperse the filler homogeneously. The films dried under reduced pressure are termed 'PC-ESM5', 'PC-ESM10', 'PC-ESM20' and 'PC-ESM40', where the numbers indicate the percentages of ESM in the hybrids in weight percentage with respect to polymer weight. Pure PVDF is termed as 'P'. A control hybrid of PVDF and nanoclay was also prepared in a similar manner and is termed 'PC', where $4 \%$ nanoclay is present in the PDVF matrix.

\subsection{Device fabrication}

The dried nanohybrids were formed into films through a compression moulding technique. These films were used for characterization. For device preparation, the nanohybrid films were cut into $1 \times 2 \mathrm{~cm}^{2}$ sizes and covered with aluminum foil on both sides. The aluminum foil acted as conducting layers for the sample. Then, electrodes were fabricated on both sides. The assembly was then covered with polypropylene tape to avoid the triboelectric effect. The whole assembly was then encapsulated in PDMS to protect it from damage and from the external environment. Special care was taken to remove trapped air through degassing during mixing of the epoxy and hardener in a $10: 1$ ratio.

\subsection{Characterization}

$X$-ray Diffraction: a Rigaku Miniflex 600 X-ray diffractometer was used to obtain the diffraction patterns at a voltage of $40 \mathrm{kV}$ and current of $15 \mathrm{~mA}$ using $\mathrm{Cu} \mathrm{K} \alpha$ radiation $(\lambda=1.54 \AA)$ at a scan rate of $3^{\circ} \mathrm{min}^{-1}$. Fourier Transform Infrared Spectroscopy (FTIR): FTIR was performed at room temperature from $650 \mathrm{~cm}^{-1}$ to $4000 \mathrm{~cm}^{-1}$ using a Nicolet 5700 instrument with $4 \mathrm{~cm}^{-1}$ resolution in ATR mode. Scanning Electron Microscopy (SEM): the surface morphologies of the samples were determined using SEM (SUPRA 40, Zeiss). Each sample was gold-coated before SEM observation. EDX spectra were recorded for elemental analysis. Polarized optical microscopy: a Leica polarized optical microscope was used to record optical images under crossed Nicols. Thin film samples $(\sim 40 \mu \mathrm{m})$ were prepared for imaging. Atomic Force Microscopy (AFM): AFM was performed to determine the changes in the surface morphologies, piezo-responses and phase changes of the samples in PFM (piezo force microscopy) mode using an NTEGRA Prima instrument (NTMDT). A DC voltage of $10 \mathrm{~V}$ was applied for the measurements to understand the piezo and nonpiezo domains in the specimens. Power measurement: the output voltage from the devices under mechanical load was measured under different resistances for power calculation, and the power was calculated using the relation $P=V^{2} / R$. A digital storage oscilloscope (TBS1072B) was used for voltage measurements.

\subsection{Biocompatibility study}

Cell viability. HeLa human cells were seeded in a culture flask, containing Dulbecco's modified Eagle's medium (DMEM) supplemented with $10 \%$ heat-inactivated fetal bovine serum (Himedia), $100 \mathrm{U} \mathrm{ml}{ }^{-1}$ penicillin and $100 \mathrm{mg} \mathrm{ml}^{-1}$ 
streptomycin. The culture flask was maintained in a $5 \% \mathrm{CO}_{2}$ incubator at $37^{\circ} \mathrm{C}$. The cytocompatibility of a material is highly desired for its biomedical application. The MTT (3-(4,5dimethylthiazol-2-yl)-2,5-diphenyltetrazolium bromide) assay was used to quantify the cytocompatibilities of the samples in terms of the percentage of cell viability. ${ }^{35}$ On $70 \%$ to $90 \%$ confluence of cells in the culture flask, the cells were trypsinized to detach them from the flask surface. After making suspensions of cells in fresh media, the cells were seeded in the wells of a 96-well culture plate and incubated at $37{ }^{\circ} \mathrm{C}$ in a $5 \% \mathrm{CO}_{2}$ incubator for 24 hours. The next day, a sterilized sample $(50 \mu \mathrm{g}$ $\mathrm{ml}^{-1}$ ) of medium was used to replace the old medium, and the culture plates were maintained in the $5 \% \mathrm{CO}_{2}$ incubator at $37^{\circ} \mathrm{C}$ for 24, 48 and 72 hours. After incubation, $100 \mu \mathrm{l}$ of the MTT solution was added and incubated. After 4 hours, dimethyl sulfoxide (DMSO) was added, and the absorbance was measured at $570 \mathrm{~nm}$. The percentage of cell viability was measured with respect to the control using the following equation.

$$
\% \text { of cell viability }=\frac{\mathrm{OD} \text { of sample }}{\mathrm{OD} \text { of control }} \times 100
$$

Cell adhesion study. For the cell adhesion study, thin coatings of the samples were made on the wells of the 96-well culture plate, and the samples were treated with DMEM for 24 hours. Next day, $1 \times 10^{4}$ HeLa cells were seeded on the surfaces of the samples, which were incubated at $37{ }^{\circ} \mathrm{C}$ in the $\mathrm{CO}_{2}$ incubator for 24 hours. The samples were washed twice with phosphate buffer saline (PBS, $\mathrm{pH}=\sim 7.4$ ) after incubation, and the phase contrast images of the cells were captured using an inverted microscope.

Fluorescence imaging. The effects of loading nanoparticles into the polymer matrix on cell proliferation were studied using fluorescence microscopy. $1 \times 10^{4}$ cells were cultured in the wells of a 96-well culture plate and were incubated for 24 hours in the $5 \% \mathrm{CO}_{2}$ incubator at $37^{\circ} \mathrm{C}$. After 24 hours of incubation, the medium was replaced by a sample of fresh medium and the culture plates were placed in the $\mathrm{CO}_{2}$ incubator at $37^{\circ} \mathrm{C}$ for 24 , 48, and 72 hours. After incubation, the cells were washed twice with PBS ( $\mathrm{pH}=\sim 7.4$ ) and were stained with acridine orange and ethidium bromide. A fluorescence microscope (Leica) was used to capture fluorescence images of the cells.

\section{Results and discussion}

\subsection{Induced structure and morphology}

The X-ray diffraction (XRD) patterns of pure PVDF and its nanohybrids with ESM (40 wt\%) and nanoclay are shown in Fig. 1a. Pure $\alpha$-phase peaks appear at the $17.6^{\circ}(100), 18.3^{\circ}(020)$ and $19.9^{\circ}$ (110) planes in pure PVDF, ${ }^{22,36,37}$ and similar peak positions were observed in the P-ESM hybrid. Moreover, the crystalline $\beta$-phase peak at $\sim 20.5^{\circ}$ (corresponding to the 200 / 110 planes) was noted in the spectrum of the PC-ESM nanohybrid. There is a clear phase change from $\alpha$ to $\beta$ in the presence of nanoclay (nanoclay-induced phase transformation), arising from the intimate interactions between the nanoclay and the
PVDF chains, ${ }^{11,38}$ while ESM alone was unable to induce $\beta$-phase in the polymer. It should be mentioned that ESM shows $\sim 40 \%$ crystalline phase (ESI Fig. S1 $\dagger$ ) and is known to be piezoelectric. ${ }^{39}$ The amounts of piezoelectric $\beta$-phase in the hybrids were calculated through the deconvolution of the XRD peaks (Fig. 1b), and the percentage $\beta$-phase fractions of various nanohybrids as a function of the ESM content are shown in Fig. 1c. PC shows a minimum piezoelectric $\beta$-phase $(32 \%)$; meanwhile, a similar quantity of nanoclay induced large amounts of $\beta$-phase in the three-component nanohybrids $(82 \%$ $\beta$-phase using $40 \% \mathrm{ESM}$ ), with a gradual enhancement in the piezoelectric phase with ESM content. It is worth mentioning that ESM alone cannot induce piezoelectricity in the PVDF matrix, while in the presence of 2-D nanoclay, high piezoelectricity in induced in the matrix polymer. The gradual change in the structure of the nanohybrids is presented in ESI Fig. S2a and b. $\dagger$ The structural alterations were also verified using FTIR spectroscopy with the presence of peaks at 836, 880 and $1167 \mathrm{~cm}^{-1}$, assigned to $\beta$-phase, in the nanohybrids (PC, PCESM) against the pure $\alpha$-peaks at 761, 797, 869, 974 and $1146 \mathrm{~cm}^{-1}$ in PVDF and P-ESM (Fig. 1d). ${ }^{11,40}$ It is interesting to note that the $\alpha$-phase peak intensity of pure PVDF considerably decreased in P-ESM, although there was no $\beta$-phase peak; this indicates decreased crystallinity in P-ESM, presumably due to the greater interaction between PVDF and ESM. The interaction was further visualized from the presence of carbonyl, amide and $-\mathrm{OH}$ groups in ESM (ESI Fig. S3 $\dagger$ ), which is responsible for greater interactions through hydrogen bonding and dipoledipole interactions. However, the presence of ESM enhances the piezoelectric $\beta$-phase significantly in the three-component nanohybrids, and therefore is suitable for energy harvesting applications.

Structural changes are often associated with the transformation in surface morphology. The polarized optical microscope images of pure PVDF and the nanohybrids are shown in Fig. 2a. Pure PVDF shows a spherulitic pattern, indicative of $\alpha$-phase, with an average diameter of $200 \mu \mathrm{m}$; meanwhile, tiny spherulite $(50 \mu \mathrm{m})$ along with a fibrous morphology is observed in P-ESM in the presence of $40 \mathrm{wt} \%$ ESM. In contrast, a mesh-like morphology is evident in PC-ESM, which is a clear indication of $\beta$-phase in the presence of nanoclay and ESM. It should be mentioned that a fibrous morphology in observed in pure ESM (ESI Fig. S4 $\dagger$ ). A similar change of morphology was also observed through SEM; large and small spherulites are evident in P and P-ESM, respectively, while the needle-like $\beta$-phase morphology is obvious in PC-ESM in the presence of nanoclay and ESM (Fig. 2b). However, the fibrous morphology of ESM along with $\beta$-nucleating 2-D layered silicate aids the growth of piezoelectric $\beta$-phase in large quantities. The enhancement of the piezoelectric phase in the threecomponent nanohybrids was also verified through piezo force microscopy. The changes in the piezo-response and phases indicate the presence of varying quantities of piezo phase domains upon the application of a DC voltage of $10 \mathrm{~V}$ (Fig. 2c). The phase angle corresponding to the line profiles of three different specimens clearly demonstrates significant changes in the phase behavior of PC-ESM compared to that of pure PVDF or 


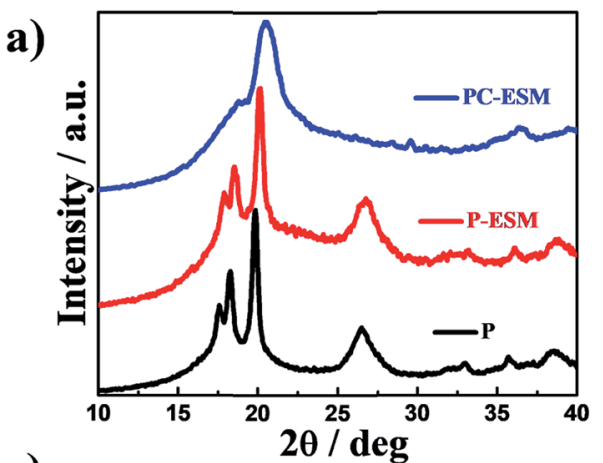

c)

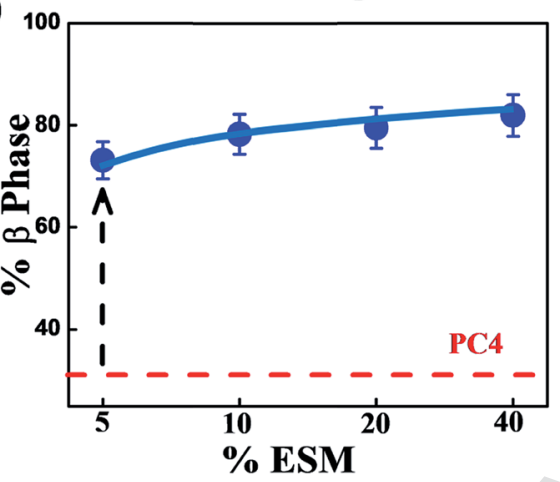

b)

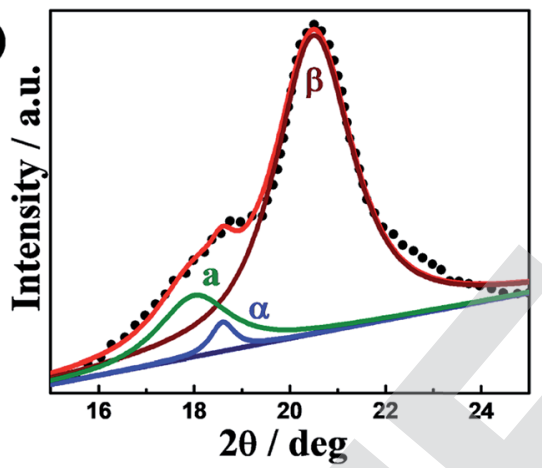

d)

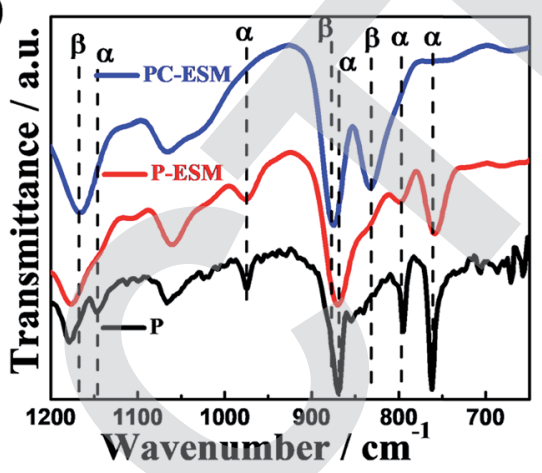

Fig. 1 (a) XRD patterns of pure PVDF, P-ESM40 and PC-ESM40 nanohybrids, showing the changes in their structure; (b) deconvolution of the XRD pattern of PC-ESM40 for the calculation of the phase fraction; (c) plot of $\beta$-phase fraction with ESM content, showing higher $\beta$-phase in PCESM. The dashed line indicates $\beta$-phase for PC and the vertical arrow shows the increase in the piezo-phase content. (d) FTIR spectra of pure PVDF, P-ESM40 and PC-ESM40 nanohybrids indicating the peak positions of the different phases.

the P-ESM hybrid (Fig. 2d). The changes in other piezoresponses are presented in ESI Fig. S5. $\uparrow$ It is worth mentioning that the application of potential causes the development of strain in the piezo specimens (because PVDF and ESM both have negative piezoelectric coefficients), as is evident from the considerable differences in the profiles before and after applying potential to the samples (ESI Fig. S6 $\dagger$ ). However, a larger piezo domain is evident in PCESM vis-à-vis pure PVDF or the P-ESM hybrid. In this juncture, it should be mentioned that ESM (piezo filler) distributed in the PVDF matrix cannot induce piezo phase in polymer matrix; meanwhile, it induces a large quantity of piezo phase in association with another 2-D nanoclay and converts most of the matrix into piezo phase, as shown in the cartoon in Fig. 2e, commensurate with the XRD and FTIR results; this leads to the development of two types of piezoelectric materials, with piezo filler in a non-piezo matrix (PESM) and piezo filler in a piezo matrix (PC-ESM), which are expected to exhibit very different energy harvesting behaviors.

\subsection{Energy harvesting using nanohybrids}

Because the nanohybrids exhibits very high piezoelectric phase, they should demonstrate energy harvesting capability. Nanogenerators were fabricated for energy harvesting using a suitable layer-by-layer assembly of an active piezoelectric material electrode and a stable coating, as shown in Fig. 3a. A rectangular-shaped electroactive hybrid material electrode was attached with aluminum foil on both sides, followed by wrapping of the whole device with poly(dimethyl siloxane). Fig. 3b shows the open circuit voltage (OCV) arising from the devices made of pure PVDF and the nanohybrids with ESM and nanoclay plus ESM under finger tapping with a frequency of $\sim 5 \mathrm{~Hz}$. The three-component nanohybrid (PC-ESM) exhibits the maximum peak-to-peak open circuit voltage of $56 \mathrm{~V}$ compared to the meager 4 and $33 \mathrm{~V}$ shown by the devices using pure PVDF and P-ESM, respectively. The estimated force from finger tapping is calculated to be around $40 \mathrm{kPa}$ (details are given in the ESI, Note S1 $\dagger$ ). The device containing the two-component hybrid (P-ESM) shows strong ESM content variation under similar strain application using finger tapping (Fig. 3c). The OCV increased systematically with ESM content and reached $35 \mathrm{~V}$ using $40 \mathrm{wt} \%$ ESM. Interestingly, the three-component nanohybrids (PC-ESM) displayed higher OCVs compared to the two-component hybrid (P-ESM) with similar ESM loading; $56 \mathrm{~V}$ was obtained using $40 \mathrm{wt} \%$ ESM (Fig. 3d), presumably due to the higher amount of piezoelectric $\beta$-phase in the nanohybrid vis-à-vis P-ESM.

Now, it is pertinent to quantify the performance of the nanogenerator on the basis of output power density. The output power is calculated by measuring the voltage at different resistances because the power depends on the external load applied on the system. The open circuit voltage increases initially with increasing resistance and then saturates (ESI Fig. S7†). The power density is calculated using the equation: 
a)

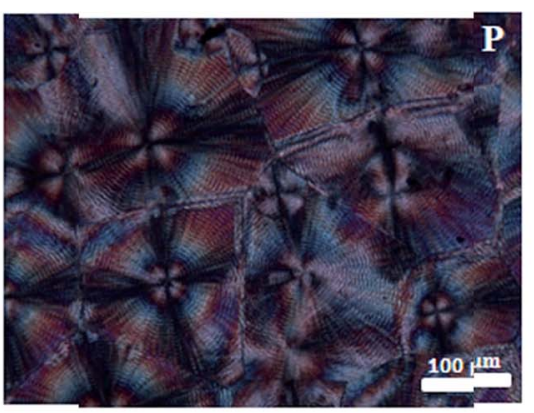

b)

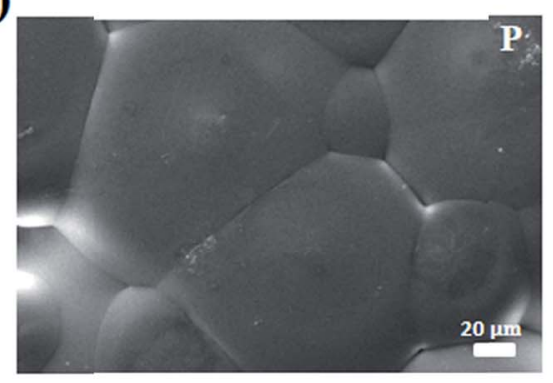

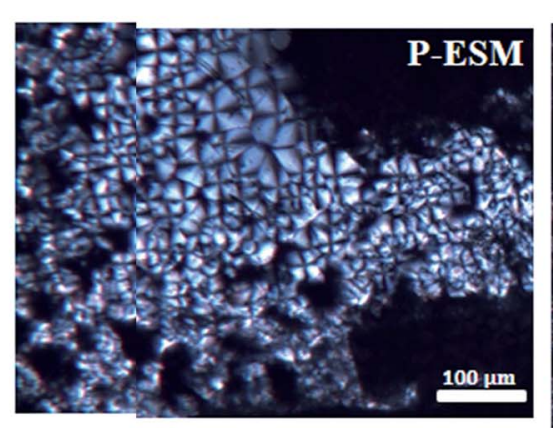

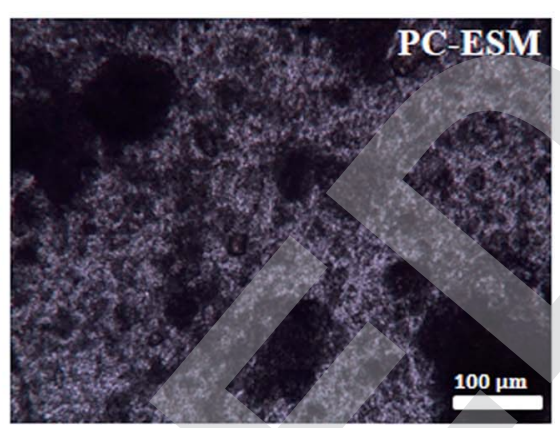

c)

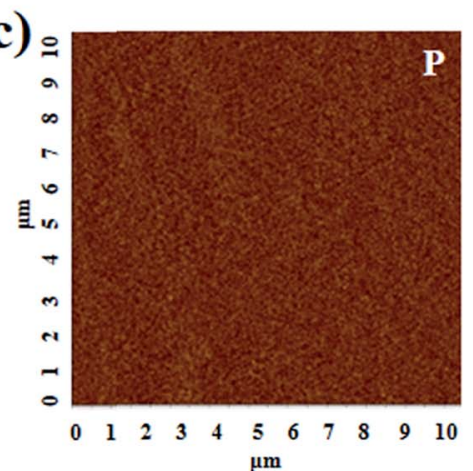

d)
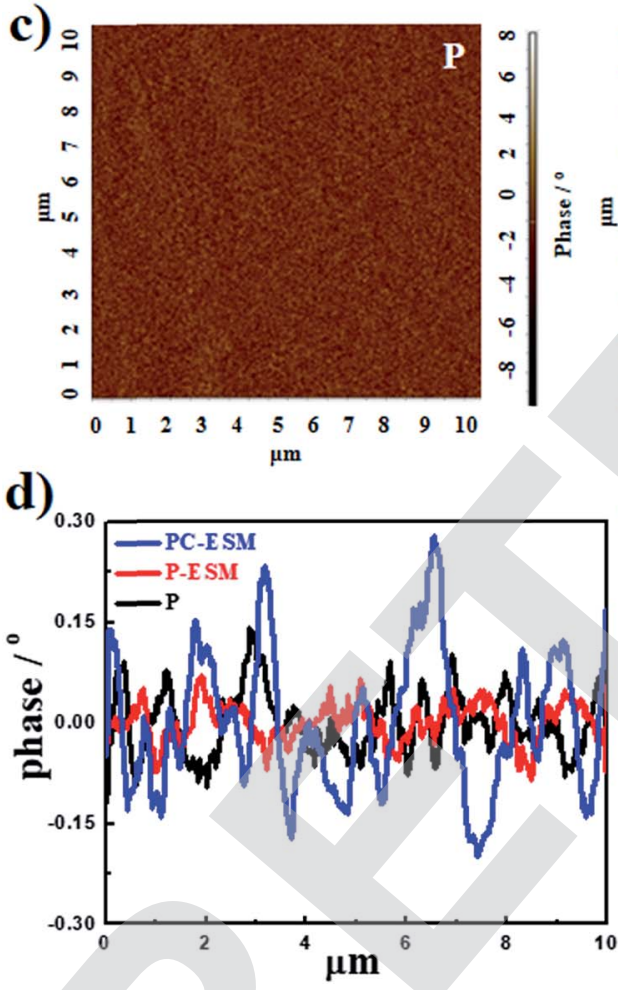
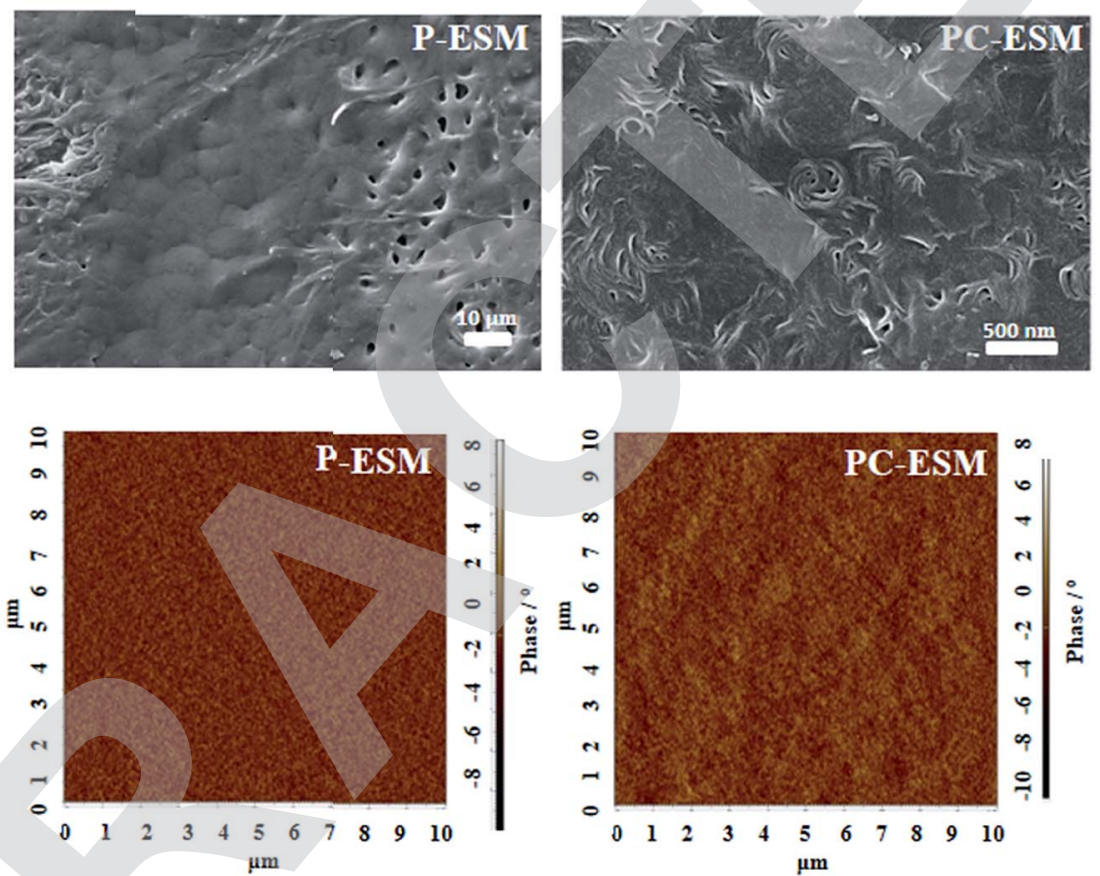

e)
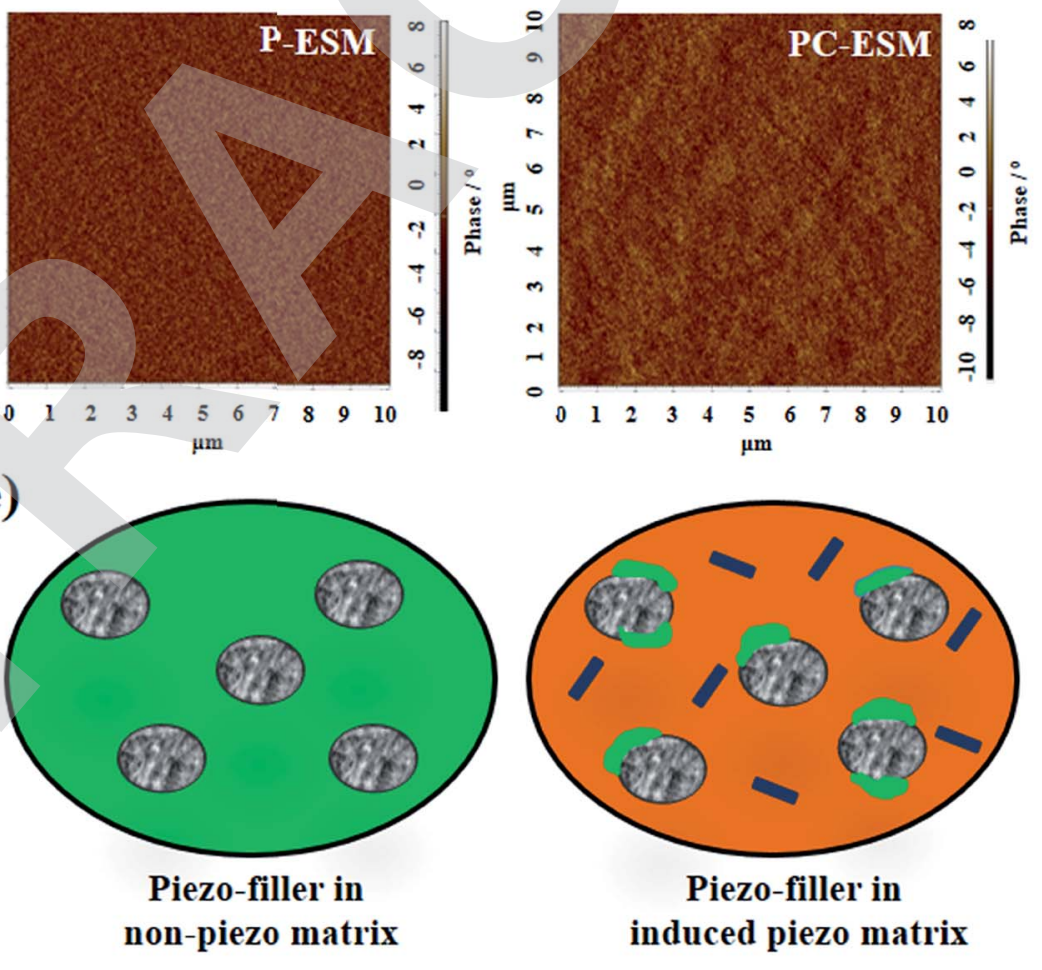

Fig. 2 (a) Polarized optical images of the indicated specimens showing spherulite in P and P-ESM and the absence of spherulite in PC-ESM. (b) Scanning electron microscope images of pure PVDF and the P-ESM and PC-ESM nanohybrids. (c) Piezo force microscopic images of pure PVDF and the P-ESM40 and PC-ESM40 nanohybrids showing better phase development in PC-ESM. (d) Phase profiles of the indicated specimens obtained from the PFM images. (e) Cartoon showing the dispersion of piezoelectric filler (grey) in the non-piezo (green) and induced piezo matrix (orange) in the absence and presence of nanoclay, respectively.

$$
P=\frac{V^{2}}{R \times A}
$$

where $V$ is the voltage across the resistance $R$ and $A$ is the area of the active device. Interestingly, the maximum power density of the PC-ESM40 nanohybrid is $55 \mu \mathrm{W} \mathrm{cm}{ }^{-2}$ (Fig. 3e), which is the highest power output value obtained using bio-waste material for energy harvesting to date. As per our knowledge, the values of output voltage and power from different bio-based nanogenerators are presented in ESI Table $\mathrm{S} 1 \dagger$ and compared with this work; PC-ESM definitely exhibits the highest power output 
a)

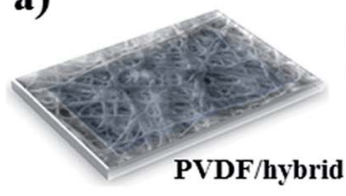

b)

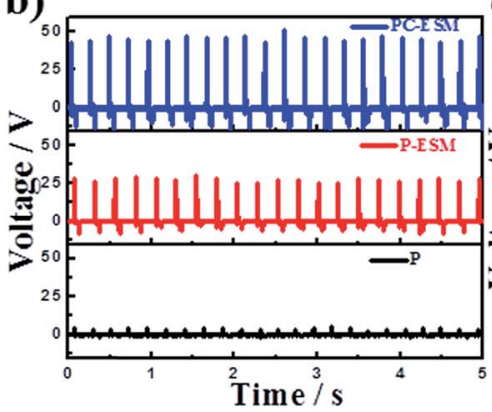

e)

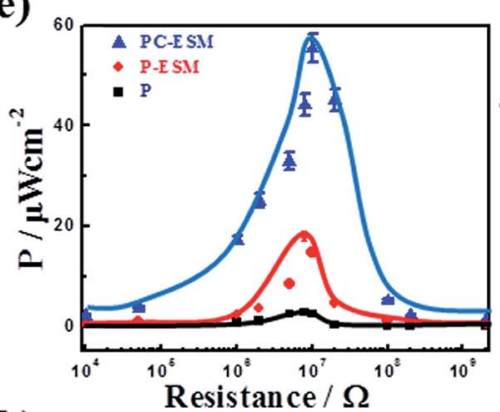

h)

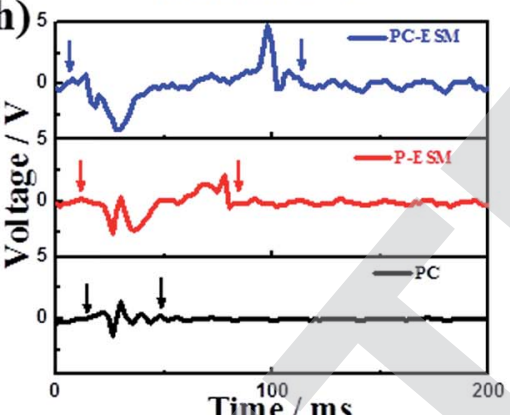

j)

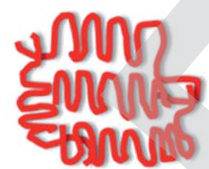

$\alpha$-phase Non-piezoelectric

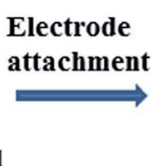

c)

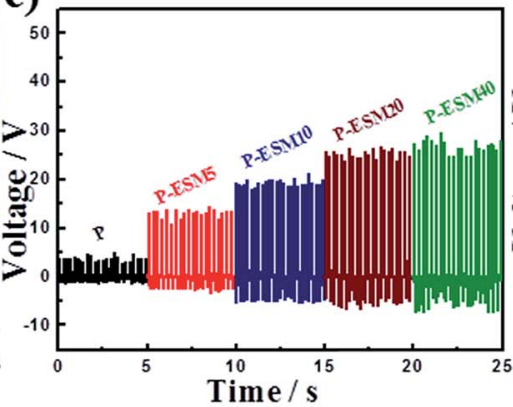

f)

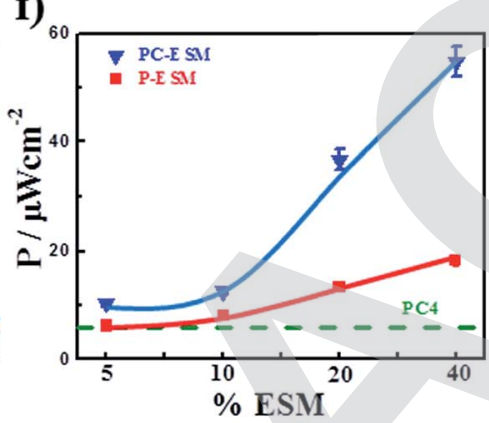

i)
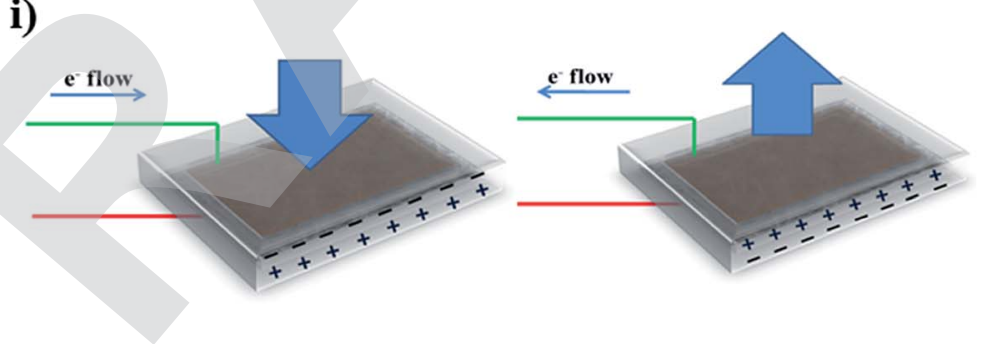

k)

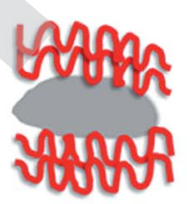

$\alpha$-phase

Less-piezoelectric

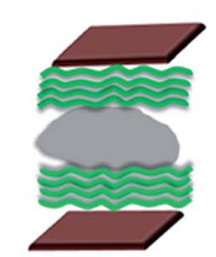

More $\beta$-phase Highly piezoelectric

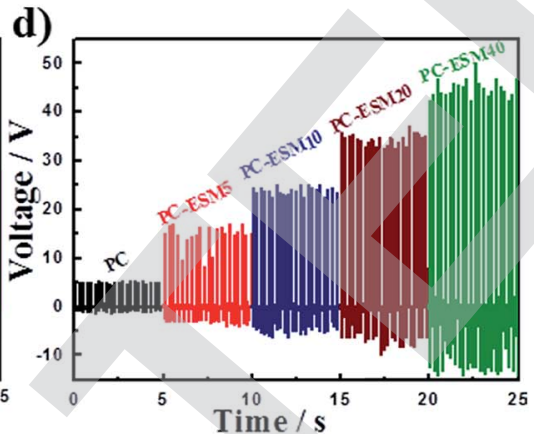

g)
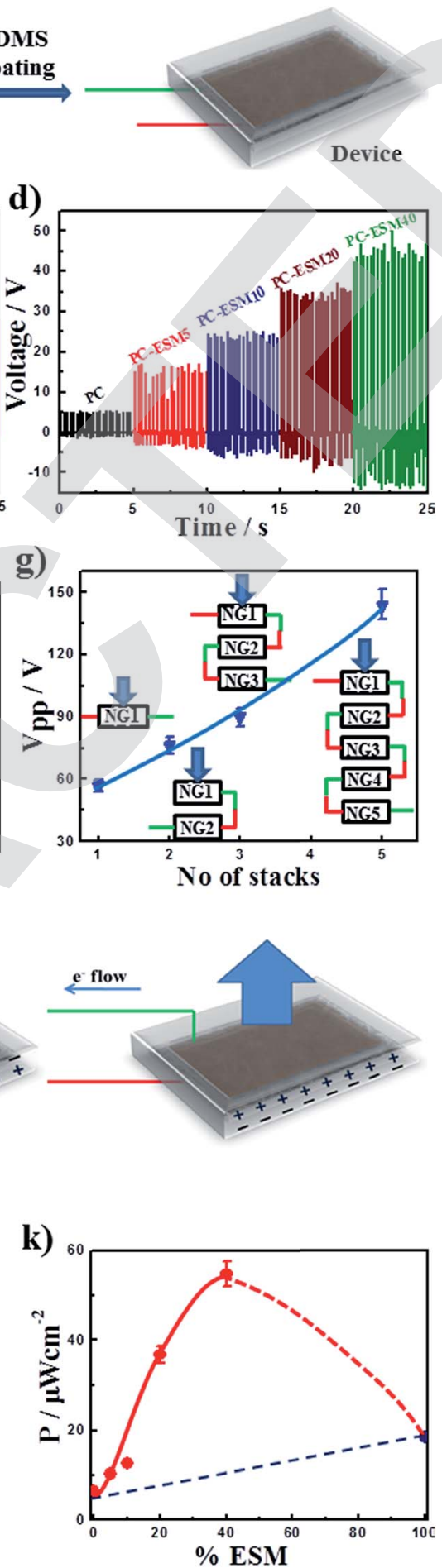

Fig. 3 (a) Layer assembly steps for the fabrication of the nanogenerator devices using pure PVDF and the nanohybrids; (b) open circuit voltage from the devices using pure PVDF, P-ESM and PC-ESM nanohybrids with 40 wt\% ESM. (c) Output OCVs from the devices fabricated using the indicated ESM contents in P-ESM. (d) Output OCVs from the devices fabricated using the indicated ESM contents in PC-ESM. (e) Variations in power density with resistance in devices fabricated with pure PVDF, P-ESM and PC-ESM nanohybrids with 40 wt\% ESM content. (f) Power density variations with ESM content from the devices made with P-ESM and PC-ESM nanohybrids. The dashed line indicates the power output value of the device made with PC (PVDF and nanoclay). (g) Open circuit voltages from the devices fabricated with the PC-ESM (40 wt\% ESM content) nanogenerator with increasing number of stacks as indicated. (h) Voltage output from the indicated unimorphs using PVDF and its nanohybrids. (i) The development of charges under two different types of mechanical force (applied and retractive force) leading to the formation of an alternating voltage - working mechanism for charge generation upon application of stress. (j) Schematics showing local ordering in the different nanohybrids, demonstrating the induced structure in the presence of ESM alone and the combined effects of ESM and nanoclay. (k) Power output from the device as a function of ESM content in the PC-ESM nanohybrid, demonstrating the synergism of nanoclay and ESM, which exhibits significantly high output. 
to date. Fig. $3 \mathrm{f}$ shows the enhancement in power density from the devices using both the nanohybrids with increasing ESM content, exhibiting systematic improvement of the power density; meanwhile, the PC-ESM devices show significantly higher power output vis-à-vis P-ESM, mainly because of the higher piezoelectric content ( $\beta$-phase) in the presence of both ESM and nanoclay. The increase in power density of the nanohybrids is significant compared to the PVDF-nanoclay composite alone (indicated by the dashed line in Fig. 3f). To check the commercial viability of the device, multiple devices (2, 3 and 5 units) were assembled in series, and their corresponding output voltages are presented in Fig. $3 g$ under similar finger tapping as before; a gradual increase of output voltage can be observed with increasing number of stacks, with very high $(144 \mathrm{~V})$ output and $100 \mu \mathrm{W} \mathrm{cm}{ }^{-2}$ for 5 units of the device stacked in series. Thus, the output voltage of the device can be amplified to the desired value by assembling multiple devices and it is thus more feasible for use in large scale industrial applications. Fig. 3 h shows the signals (output voltage) from different nanogenerator unimorphs under a single compression and releasing cycle. The starting and end points of the voltage generation are indicated by down arrows, suggesting a high response time $(100 \mathrm{~ms})$ for the device made with PC-ESM against $30 \mathrm{~ms}$ for PC. It should be mentioned that the response time indicates the duration of power generation from the device under mechanical stress. Another intriguing feature of the nanohybrid device is that there is a considerable time gap between the compress and release modes of power generation which is higher for PC-ESM than for P-ESM; this phenomenon is not present for PC. The working principle of bio-based piezoelectric materials is still under investigation because their unusual nature cannot be explained by classical piezoelectricity theory, which is based on the ideal/perfect crystalline structure. ${ }^{41}$ The possible working mechanism of the voltage generation is explained in Fig. $3 \mathrm{i}$ by considering the physical changes (rotation of dipole) upon application of mechanical stress in the compression and release modes. Due to the floppy nature of ESM, as evident from its SEM image, the PC-ESM and P-ESM hybrids are slow relaxation systems; thus, they require relatively longer times to rotate dipoles under the two modes of force (compression and release) compared to the compact system in $\mathrm{PC}$, where no noticeable time lag is observed between the two modes. In PVDF and the nanoclay hybrid (PC), the piezoelectricity is explained through epitaxial crystallization of the PVDF on the surface of the 2-D nanoclay, which leads to the transformation of its $\alpha$-phase into electroactive $\beta$-phase; the extent of conversion dictates the piezoelectric phase. ESM has a porous structure, so it is soft and flexible; this eventually leads to more displacement than the normal compact structure experiences under a fixed load, giving rise to high piezoelectricity in ESM. ${ }^{4}$ ESM contains collagens type I, V, X and different proteins, such as osteopontin, keratin, proteoglycans and glycoproteins. The piezoelectricity in ESM is due to the combined effects of the collagen and the proteins. The polarization and piezoelectricity in type I collagen fibrils has been established to be due to the existence of $\mathrm{N}$ and $\mathrm{C}$ terminal telopeptides and $\mathrm{C} 6$ symmetry in the crystalline chains. ${ }^{29}$ Collagen fibers and different proteins are present in ESM. External compressive stress on the oriented collagen structure induces high internal friction among the hydrogen-bonded $\alpha$-helices. ${ }^{42}$ Therefore, the deformation of the triple helical structure aids the creation of dipole moments in ESM under stress. Similar mechanisms have been observed in M13 bacteriophage, ${ }^{26}$ fish scale ${ }^{\mathbf{3 4}}$ and fish swimming bladder. ${ }^{43}$ ESM contains many collagen microfibrils which generate electric dipole moments upon the application of mechanical stress. ${ }^{42}$ Hence, under applied stress, charges develop on the top and bottom of ESM due to breaking of the symmetry present in the collagen moieties. ${ }^{29,44}$ The addition of ESM to PVDF or to the PVDF-nanoclay matrix converts the system into piezo phase in the non-piezo matrix and piezo phase in the piezo matrix, respectively. The enhancement of the piezoelectric response in the nanohybrids is directly related to the mutual electromechanical interactions among the fibers (needle-like $\beta$-phase in the matrix and ESM fibrils) under external applied stress. ${ }^{\mathbf{2 9}, \mathbf{4 4}}$ There are different types of proteins in ESM; hence, there is a possibility of interconnections between them, either through hydrogen bonding or van der Waals interactions, which disrupt under mechanical stress, leading to enhanced piezoelectricity in the nanohybrids. In addition to this, the porosity of ESM plays an important role because a porous structure experiences more displacement than a compact structure under similar applied stress.,45 However, the potential difference, caused by the orientation of the electric dipole, results in electron flow from one direction to another; meanwhile, the electrons flow in the reverse direction upon release of the stress, causing alternating current in the circuit. ${ }^{46}$ Based on the varying nature of the interactions in various hybrids/nanohybrids, the location and domain sizes are presented in the form of a cartoon in Fig. 3j. Pure PVDF crystallizes only in $\alpha$-phase and does not show any piezoelectricity; meanwhile, nanoclay induces $\beta$ phase in PVDF (PC), whose overall crystallinity is lower, and thereby exhibits meager piezoelectricity. The introduction of electroactive ESM in pure PVDF (P-ESM; piezo phase in a nonpiezo matrix) shows an $\alpha$-phase PVDF matrix where piezo filler is dispersed; this system exhibits moderately high piezoelectricity in the hybrids with higher ESM content. On the other hand, good interactive three-component systems (PC-ESM) induce $\beta$-phase in the PVDF matrix along with piezo filler; this creates a near-ideal system where the whole materials exhibit piezo phase (except for a small amount of amorphous phase present in the system) showing layered orientation of three different components, namely $\beta$-phase on the surface of the 2-D nanoclay along with a layer of ESM. Currently, it is apparent that ESM increases the piezoelectricity to a limited extent only, while nanoclay and ESM enhance the piezoelectricity and subsequent power generation to a much greater value; this raises the possibility of synergism. The output power from the devices has been plotted as a function of ESM content in PC-ESM, showing strong synergism (significantly above the values predicted by the linear mixture rule, as shown by the dotted line in the figure) (Fig. 3k). This synergism is insignificant in P-ESM, presumably due to less interaction 
a)

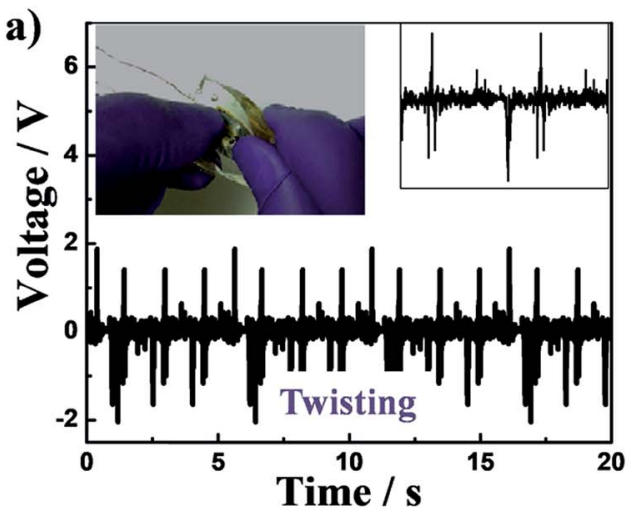

c)
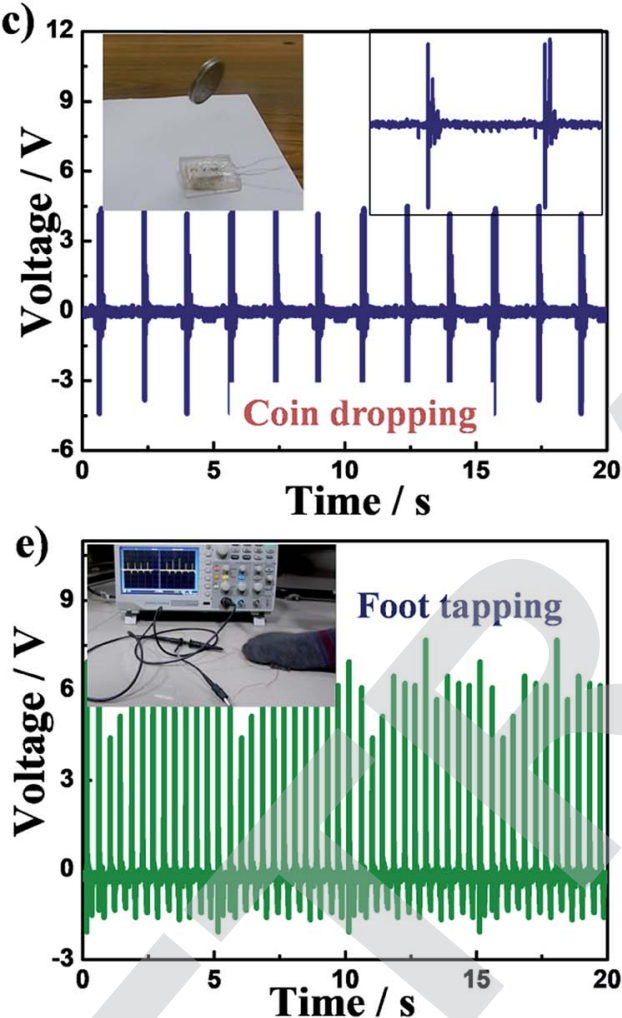

g)

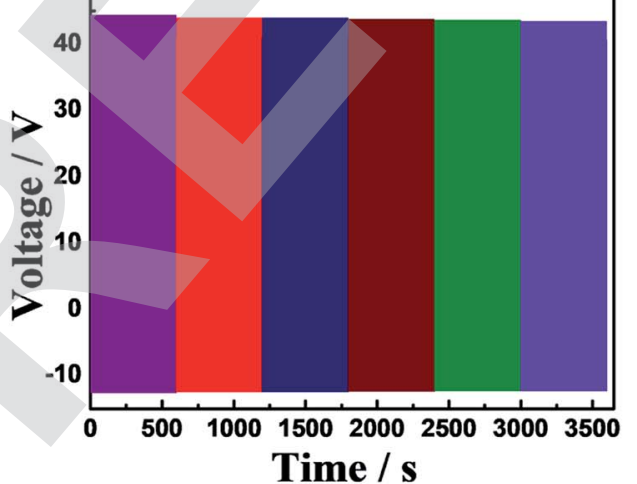

b)

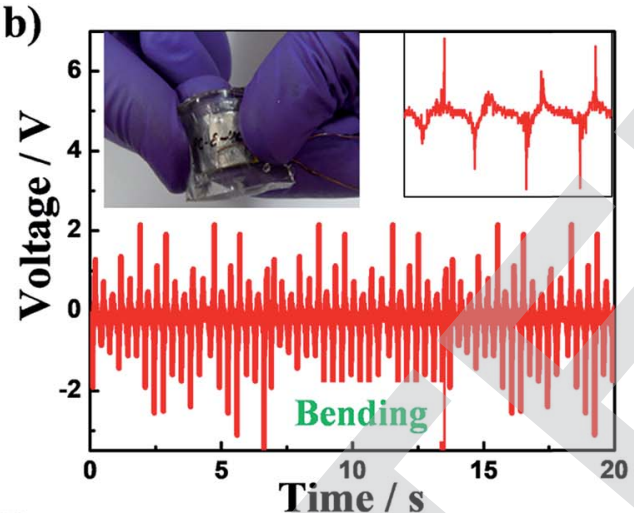

d)

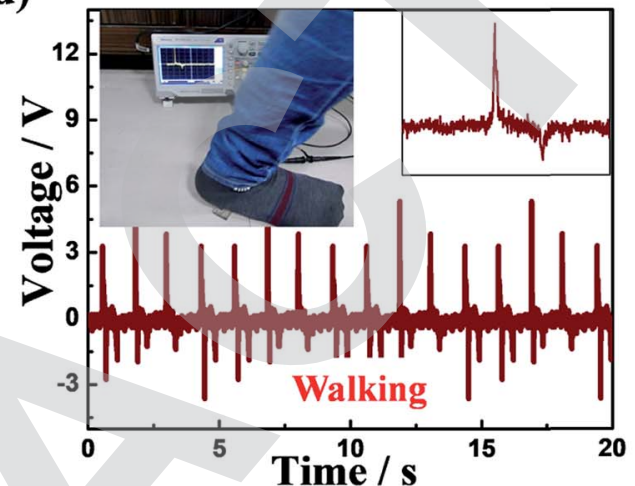

f)

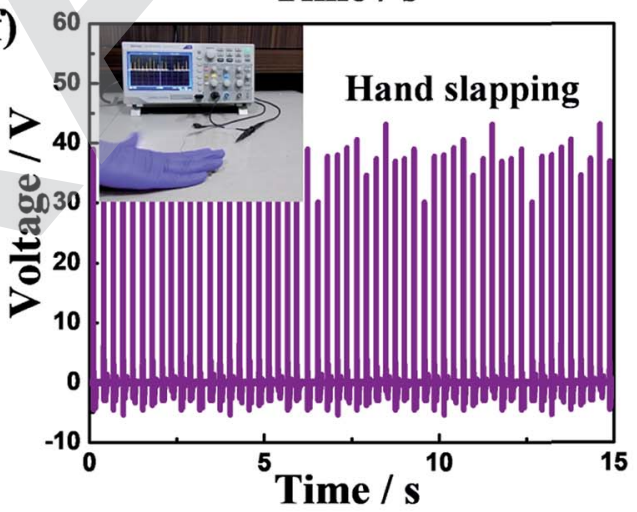

h)

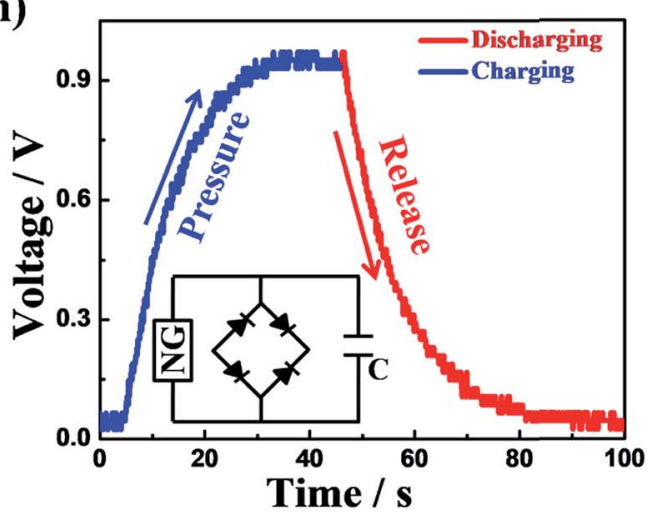

Fig. 4 Demonstration of practical applications of the nanogenerator to harvest energy by applying normal human movements, e.g. (a) twisting; (b) bending; (c) coin dropping; (d) walking; (e) foot tapping; and (f) hand slapping. (g) Mechanical and durability tests of the device after obtaining the device performance for a sufficiently long time, showing very similar output voltages; and (h) the ability of the power output from the device made with PC-ESM40 to charge a capacitor, followed by the discharging kinetics. 
between PVDF and ESM (ESI Fig. S8 $\dagger$ ). However, a very high output voltage of $56 \mathrm{~V}$ was achieved from the nanohybrid, with a maximum power density of $55 \mu \mathrm{W} \mathrm{cm} \mathrm{cm}^{-2}$ from a single piezoelectric device which increased to $144 \mathrm{~V}$ of OCV and 100 $\mu \mathrm{W} \mathrm{cm}{ }^{-2}$ from a stack containing 5 units of the device.

\subsection{Practical applications of nanogenerators}

In the preceding section, very high power generation is demonstrated using the three-component nanohybrid by applying the finger tapping method. The harvesting ability of the devices with different human activities as waste mechanical energy sources can also be verified to understand the efficacy of the energy harvesting. Fig. 4a shows the voltage output upon twisting the nanogenerator (PC-ESM), which produces a peakto-peak output voltage of $\sim 4 \mathrm{~V}$. The inset images show the force application mode and zooming of the output voltage. On bending (Fig. 4b) and coin dropping (Fig. 4c) (INR 5-rupee coin), the device produces $\sim 5.6$ and $8 \mathrm{~V}$, respectively; although the weight of the coin is lower, the impact of the freefalling coin is greater, which justifies the higher output. For more realistic application, the output voltage from the nanogenerator on walking is shown in Fig. $4 \mathrm{~d}$ as $\sim 9.5 \mathrm{~V}$; on foot tapping, the output voltage was measured to be $\sim 10 \mathrm{~V}$ (Fig. 4e), which can also light up LEDs. The hand slapping method was found to be more effective, with an output voltage of $\sim 50 \mathrm{~V}$; this is significantly higher than the other modes, primarily due to the greater impact and higher contact area (Fig. 4f). However, various body motions could also generate significant voltage for harvesting energy. The respective power densities of these human activities have also been calculated and are presented in ESI Fig. S9; $\uparrow$ the corresponding videos are shown in ESI Video S1. $\dagger$ The mechanical stability and durability tests of the device are shown in Fig. 4g, which clearly demonstrates good performance of the device even after repeated use. Now, it is pertinent to understand whether the device is able to store charge in a capacitor. The device was attached to a capacitor $(1 \mu \mathrm{F})$ through a rectifier circuit and also connected to a digital oscilloscope to read the corresponding charging and discharging voltages from the device. The hand tapping method clearly demonstrates charging of the capacitor and reaches a plateau, followed by discharging under a suitable external load (Fig. 4h). The response times for the charging and discharging phenomena were found to be 30 and 35 s, respectively. Repeated charging and discharging of the capacitor is shown in ESI Fig. S10. $\uparrow$ However, the nanogenerator made with bio-waste and nanoparticles embedded in PVDF could produce sufficient energy to be stored and utilized later, demonstrating the efficacy of the bio-inspired device for energy harvesting purposes. Using the nanogenerator, LEDs could be lit by waste mechanical stress. The video is shown in ESI Video S2. $\dagger$ From these results, a)

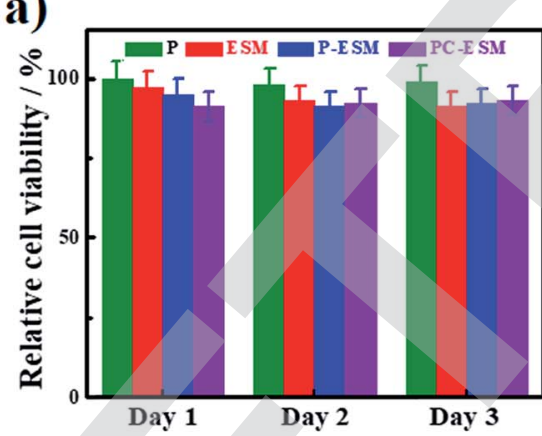

c)

Control

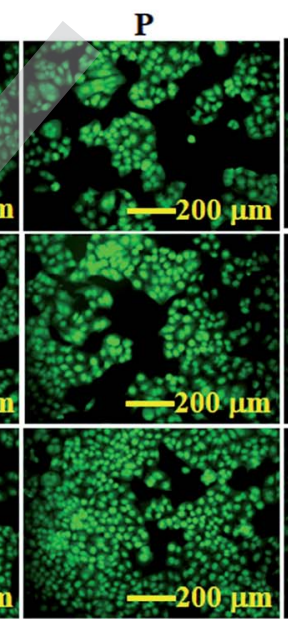

b)
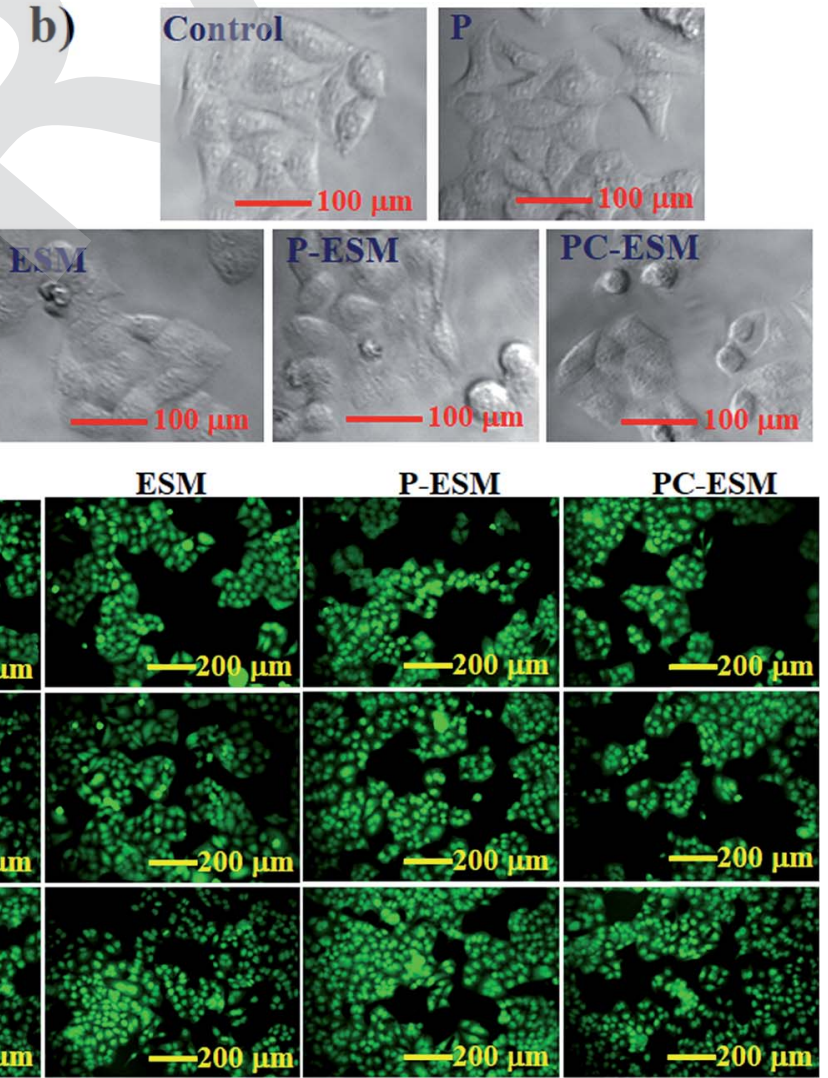

Fig. 5 (a) Cell viability as a function of time comparing pure PVDF and its hybrids; (b) phase contrast images of cells adhered on the indicated samples; and (c) fluorescence images of pure PVDF and its hybrid/nanohybrids showing their biocompatibility through cell proliferation. 
it is clear that our bio-based piezoelectric nanogenerator is efficient for realistic applications.

\subsection{Biocompatibility study}

Cytocompatibility is one of the essential parameters for the biomedical application of any material. The cytocompatibility of a material is quantified in terms of cell viability, and the MTT assay is performed to check the viable cells in the presence of different samples. Yellow tetrazolium salt is converted to red formazan derivatives by the mitochondria of viable cells through intercellular reduction in the MTT assay. The cell viability of HeLa cells in a 96-well culture plate without any material is taken as a control, and the cell viability in the presence of a sample is expressed with respect to the control. Fig. 5a shows that the cell viabilities of pure PVDF, ESM, PESM40 and PC-ESM40 are $100 \%$, and no significant changes in cell viability were observed after 48 and 72 hours. This result suggests that the developed materials help the cells to proliferate and are cytocompatible in nature. It should be mentioned that the cell viability of PC-ESM40 after 72 hours is greater than those of ESM and P-ESM40. Further, the cell adhesion over the surface of the samples was studied in terms of morphological investigation of the cells. Cell adhesion is the preliminary step which decides the subsequent steps, such as cell proliferation. The phase contrast images (Fig. 5b) show an almost spread morphology of the cells on the surface of the samples; this indicates that the cells are nicely adhered to the samples, which helps the cells to proliferate further with time, as observed in the cytotoxicity measurements. The viability of HeLa cells was further confirmed through fluorescence imaging of the HeLa cells in the presence of pure PVDF, ESM, P-ESM40 and PCESM40 (Fig. $5 \mathrm{c}$ ). ${ }^{47} \mathrm{~A}$ combination of acridine orange (AO) and ethidium bromide (EtBr) was used to stain the cells. The acridine orange binds with DNA to show green fluorescence, while the ethidium bromide shows red fluorescence. Live cells only show AO fluorescence, while apoptotic cells show both AO and $\mathrm{EtBr}$ fluorescence. Fig. $5 \mathrm{c}$ shows the number densities of HeLa cells, which are almost equal for all the samples at a particular time of cell proliferation, including the control; the cell density increases gradually with time. Hence, the developed nanohybrid materials help the cells to proliferate; thus, these materials can safely be used for biomedical applications, especially for implant devices which can generate energy utilizing waste biomechanical performance.

\section{Conclusion}

This work demonstrates a nanohybrid nanogenerator based on abundantly available bio-waste egg shell membrane (ESM) as an efficient energy harvester. Two/three-component hybrids/ nanohybrids have been synthesized by embedding nanoclay and a nanoclay-ESM combination in PVDF through a solution route. ESM does not alter the structure of PVDF, while nanoclay in the presence of ESM almost completely converts the matrix polymer into piezoelectric phase. The structural conversion was confirmed through XRD and FTIR studies and was also supported by morphological studies using optical and scanning electron microscopy. The local ordering of piezo-phase was revealed through AFM studies. Devices were fabricated using piezo-innonpiezo and piezo-in-piezo phases of hybrids/nanohybrids with various ESM contents. The optimized nanogenerator displays an open circuit voltage of $56 \mathrm{~V}$ and a maximum power density of $55 \mu \mathrm{W} \mathrm{cm}{ }^{-2}$ under finger pressing; meanwhile, upon increasing the stack number (five), the output voltage improves to $144 \mathrm{~V}$ with a maximum power density of $100 \mu \mathrm{W} \mathrm{cm}{ }^{-2}$. The performance of the device was also shown to be efficient under different types of mechanical stress and human motions, such as bending, twisting, foot tapping, and walking, which indicates its sensitive nature towards minimal load application and significantly higher power output. The underlying mechanism of the energy storing capability of the nanogenerator has been established to be the very high piezoelectric phase induced by ESM and nanoclay. The nanogenerator has the potential to light LEDs through the finger tapping mode of stress application. The device can be installed under shoe soles, trade mills, floors and any other daily used devices to power up through human activities. The nanohybrid was found to be fully biocompatible, as evident from cellular studies (cell viability, cell adhesion and cell proliferation). Because the device is biocompatible, it has the potential to be used as an energy source for self-powered implants, e-health care monitoring and in vitro/in vivo diagnostics.

\section{Conflicts of interest}

The authors declare no conflict of interest.

\section{Acknowledgements}

AG acknowledges the institute for her teaching assistantship. Authors acknowledge the DIC-IIT (BHU) project for financial assistance.

\section{References}

1 Z. L. Wang and J. Song, Piezoelectric Nanogenerators Based on Zinc Oxide Nanowire Arrays, Science, 2006, 312(5771), 242-246.

2 Y. Qin, X. Wang and Z. L. Wang, Microfibre-Nanowire Hybrid Structure for Energy Scavenging, Nature, 2008, 451(7180), 809-813.

3 R. Yang, Y. Qin, L. Dai and Z. L. Wang, Power Generation with Laterally Packaged Piezoelectric Fine Wires, Nat. Nanotechnol., 2009, 4(1), 34-39.

4 Y. Mao, P. Zhao, G. McConohy, H. Yang, Y. Tong and X. Wang, Sponge-like Piezoelectric Polymer Films for Scalable and Integratable Nanogenerators and Self-powered Electronic Systems, Adv. Energy Mater., 2014, 4(7), 1301624.

5 S. Wang, Z. L. Wang and Y. Yang, A One-Structure-Based Hybridized Nanogenerator for Scavenging Mechanical and Thermal Energies by Triboelectric-PiezoelectricPyroelectric Effects, Adv. Mater., 2016, 28(15), 2881-2887. 
6 C. Sun, J. Shi, D. J. Bayerl and X. Wang, PVDF Microbelts for Harvesting Energy from Respiration, Energy Environ. Sci., 2011, 4(11), 4508-4512.

7 S. K. Karan, R. Bera, S. Paria, A. K. Das, S. Maiti, A. Maitra and B. B. Khatua, An Approach to Design Highly Durable Piezoelectric Nanogenerator Based on Self-Poled PVDF/AlOrGO Flexible Nanocomposite with High Power Density and Energy Conversion Efficiency, Adv. Energy Mater., 2016, 6(20), 1601016.

8 J. Wang, S. Li, F. Yi, Y. Zi, J. Lin, X. Wang, Y. Xu and Z. L. Wang, Sustainably Powering Wearable Electronics Solely by Biomechanical Energy, Nat. Commun., 2016, 7, 12744.

9 N. R. Alluri, B. Saravanakumar and S. J. Kim, Flexible, Hybrid Piezoelectric Film (BaTi $\left.(1-x) \mathrm{Zr} x \mathrm{O}_{3}\right)$ PVDF Nanogenerator as a Self-Powered Fluid Velocity Sensor, ACS Appl. Mater. Interfaces, 2015, 7(18), 9831-9840.

10 J. Chun, K. Y. Lee, C. Y. Kang, M. W. Kim, S. W. Kim and J. M. Baik, Embossed Hollow Hemisphere-based Piezoelectric Nanogenerator and Highly Responsive Pressure Sensor, Adv. Funct. Mater., 2014, 24(14), 2038-2043.

11 A. Gaur, C. Kumar, R. Shukla and P. Maiti, Induced Piezoelectricity in Poly (vinylidene fluoride) Hybrid as Efficient Energy Harvester, ChemistrySelect, 2017, 2(27), 8278-8287.

12 S. Jang, H. Kim, Y. Kim, B. J. Kang and J. H. Oh, Honeycomblike nanofiber based triboelectric nanogenerator using selfassembled electrospun poly (vinylidene fluoride-cotrifluoroethylene) nanofibers, Appl. Phys. Lett., 2016, 108(14), 143901.

13 C. Kumar, A. Gaur, S. Tiwari, A. Biswas, S. K. Rai and P. Maiti, Bio-waste polymer hybrid as induced piezoelectric material with high energy harvesting efficiency, Composites Communications, 2019, 11, 56-61.

14 S. S. Won, M. Sheldon, N. Mostovych, J. Kwak, B. S. Chang, C. W. Ahn, A. I. Kingon, I. W. Kim and S. H. Kim, Piezoelectric Poly (vinylidene fluoride trifluoroethylene) Thin Film-based Power Generators using Paper Substrates for Wearable Device Applications, Appl. Phys. Lett., 2015, 107(20), 202901.

15 J. Chen, Y. Huang, N. Zhang, H. Zou, R. Liu, C. Tao, X. Fan and Z. L. Wang, Micro-cable Structured Textile for Simultaneously Harvesting Solar and Mechanical Energy, Nat. Energy, 2016, 1(10), 16138.

16 S. Tiwari, A. Gaur, C. Kumar and P. Maiti, Enhanced piezoelectric response in nanoclay induced electrospun PVDF nanofibers for energy harvesting, Energy, 2019, 171, 485-492.

17 S. Y. Chung, S. Kim, J.-H. Lee, K. Kim, S.-W. Kim, C.-Y. Kang, S.-J. Yoon and Y. S. Kim, All-solution-processed Flexible Thin Film Piezoelectric Nanogenerator, Adv. Mater., 2012, 24(45), 6022-6027.

18 K. I. Park, C. K. Jeong, J. Ryu, G. T. Hwang and K. J. Lee, Flexible and Large-Area Nanocomposite Generators Based on Lead Zirconate Titanate Particles and Carbon Nanotubes, Adv. Energy Mater., 2013, 3(12), 1539-1544.
19 J. Yan and Y. G. Jeong, High Performance Flexible Piezoelectric Nanogenerators Based on $\mathrm{BaTiO}_{3}$ Nanofibers in Different Alignment Modes, ACS Appl. Mater. Interfaces, 2016, 8(24), 15700-15709.

20 A. Koka and H. A. Sodano, A low-frequency Energy Harvester from Ultralong, Vertically Aligned $\mathrm{BaTiO}_{3}$ Nanowire Arrays, Adv. Energy Mater., 2014, 4(11), 1301660.

21 C. Kumar, A. Gaur, S. K. Rai and P. Maiti, Piezo devices using poly(vinylidene fluoride)/reduced graphene oxide hybrid for energy harvesting, Nano-Struct. Nano-Objects, 2017, 12, 174181.

22 A. Gaur, R. Shukla, B. Kumar, A. Pal, S. Chatterji, R. Ranjan and P. Maiti, Processing and Nanoclay Induced Piezoelectricity in Poly (vinylidene fluoride-co-hexafluoro propylene) Nanohybrid for Device Application, Polymer, 2016, 97, 362-369.

23 R. A. Whiter, V. Narayan and S. Kar-Narayan, A Scalable Nanogenerator Based on Self-poled Piezoelectric Polymer Nanowires with High Energy Conversion Efficiency, Adv. Energy Mater., 2014, 4(18), 1400519.

24 S. Paria, S. K. Karan, R. Bera, A. K. Das, A. Maitra and B. B. Khatua, A Facile Approach to Develop a Highly Stretchable $\mathrm{PVC} / \mathrm{ZnSnO}_{3}$ Piezoelectric Nanogenerator with High Output Power Generation for Powering Portable Electronic Devices, Ind. Eng. Chem. Res., 2016, 55(40), 10671-10680.

25 W. Tang, J. Tian, Q. Zheng, L. Yan, J. Wang, Z. Li and Z. L. Wang, Implantable Self-powered Low-level Laser Cure System for Mouse Embryonic Osteoblasts' Proliferation and Differentiation, ACS Nano, 2015, 9(8), 7867-7873.

26 B. Y. Lee, J. Zhang, C. Zueger, W. Chung, S. Y. Yoo, E. Wang, J. Meyer, R. Ramesh and S. W. Lee, Virus-based Piezoelectric Energy Generation, Nat. Nanotechnol., 2012, 7(6), 351-356.

27 A. A. Gandhi, M. Wojtas, S. B. Lang, A. L. Kholkin and S. A. M. Tofail, Piezoelectricity in Poled Hydroxyapatite Ceramics, J. Am. Ceram. Soc., 2014, 97(9), 2867-2872.

28 E. Fukada and I. Yasuda, Piezoelectric Effects in Collagen, Jpn. J. Appl. Phys., 1964, 3(2), 117.

29 M. Minary-Jolandan and M. Yu, Nanoscale Characterization of Isolated Individual Type I Collagen Fibrils: Polarization and Piezoelectricity, Nanotechnology, 2009, 20(8), 085706.

30 M. M. Alam and D. Mandal, Native Cellulose Microfiberbased Hybrid Piezoelectric Generator for Mechanical Energy Harvesting Utility, ACS Appl. Mater. Interfaces, 2016, 8, 1555-1558.

31 S. K. Ghosh and D. Mandal, Bio-assembled, Piezoelectric Prawn Shell made Self-powered Wearable Sensor for Noninvasive Physiological Signal Monitoring, Appl. Phys. Lett., 2017, 110(12), 123701.

32 S. K. Ghosh and D. Mandal, High-performance Biopiezoelectric Nanogenerator made with Fish Scale, Appl. Phys. Lett., 2016, 109(10), 103701.

33 S. Maiti, S. K. Karan, J. Lee, A. K. Mishra, B. B. Khatua and J. K. Kim, Bio-waste Onion Skin as an Innovative Naturedriven Piezoelectric Material with High Energy Conversion Efficiency, Nano Energy, 2017, 42, 282-293. 
34 M. Baláž, Eggshell Membrane Biomaterial as a Platform for Applications in Materials Science, Acta Biomater., 2014, 10, 3827-3843.

35 A. Biswas, A. P. Singh, D. Rana, V. K. Aswal and P. Maiti, Biodegradable Toughened Nanohybrid Shape Memory Polymer for Smart Biomedical Applications, Nanoscale, 2018, 10(21), 9917-9934.

36 D. Shah, P. Maiti, E. Gunn, D. F. Schmidt, D. D. Jiang, C. A. Batt and E. P. Giannelis, Dramatic Enhancements in Toughness of Polyvinylidene Fluoride Nanocomposites via Nanoclay-directed Crystal Structure and Morphology, Adv. Mater., 2004, 16, 1173-1177.

37 V. K. Tiwari, P. K. Kulriya, D. K. Avasthi and P. Maiti, Poly(Vinylidene fluoride-co-hexafluoro propylene)/Layered Silicate Nanocomposites: The Effect of Swift Heavy Ion, $J$. Phys. Chem. B, 2009, 113(34), 11632-11641.

38 V. K. Tiwari, T. Shripathi, N. P. Lalla and P. Maiti, Nanoparticle induced piezoelectric, super toughened, radiation resistant, multi-functional nanohybrids, Nanoscale, 2012, 4(1), 167-175.

39 S. K. Karan, S. Maiti, S. Paria, A. Maitra, S. K. Si, J. K. Kim and B. B. Khatua, A New Insight towards Eggshell Membrane as High Energy Conversion Efficient Bio-piezoelectric Energy Harvester, Materials Today Energy, 2018, 9, 114-125.

40 A. Gaur, C. Kumar, S. Tiwari and P. Maiti, Efficient Energy Harvesting using Processed Poly (vinylidene fluoride) Nanogenerator, ACS Appl. Energy Mater., 2018, 1, 3019-3024.
41 E. Fukada, Poiseuille Medal Award Lecture: Piezoelectricity of Biopolymers, Biorheology, 1995, 32(6), 593-609.

42 S. M. Yu, Piezoelectric Devices: Squeezed Virus Produces Electricity, Nat. Nanotechnol., 2012, 7(6), 343.

43 S. K. Ghosh and D. Mandal, Efficient Natural Piezoelectric Nanogenerator: Electricity Generation from Fish Swim Bladder, Nano Energy, 2016, 28, 356-365.

44 L. Persano, C. Dagdeviren, C. Maruccio, L. De Lorenzis and D. Pisignano, Cooperativity in the Enhanced Piezoelectric Response of Polymer Nanowires, Adv. Mater., 2014, 26, 7574-7580.

45 S. Cha, S. M. Kim, H. Kim, J. Ku, J. I. Sohn, Y. J. Park, B. G. Song, M. H. Jung, E. K. Lee and B. L. Choi, Porous PVDF as Effective Sonic Wave Driven Nanogenerators, Nano Lett., 2011, 11, 5142-5147.

46 J. H. Lee, H. J. Yoon, T. Y. Kim, M. K. Gupta, J. H. Lee, W. Seung, H. Ryu and S. W. Kim, Micropatterned P(VDF-TrFE) Film-Based Piezoelectric Nanogenerators for Highly Sensitive Self-Powered Pressure Sensors, Adv. Funct. Mater., 2015, 25, 3203-3209.

47 A. Biswas, M. Amarajeewa, S. Senapati, M. Sahu and P. Maiti, Sustained Release of Herbal Drugs using Biodegradable Scaffold for Faster Wound Healing and Better Patient Compliance, Nanomedicine, 2018, 14(7), 2131-2141. 